\title{
NURBS Curves in Parametric Integral Equations System for Modeling and Solving Boundary Value Problems in Elasticity
}

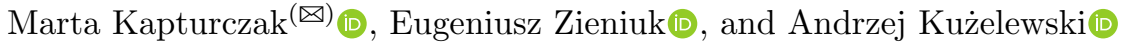 \\ Institute of Informatics, University of Bialystok, Białystok, Poland \\ \{mkapturczak, ezieniuk\}@ii.uwb.edu.pl
}

\begin{abstract}
This paper presents a way to improve the boundary shape modeling process in solving boundary value problems in elasticity. The inclusion of NURBS curves into the mathematical formalism of the parametric integral equations system method (PIES) is proposed. The advantages of such an application are widely discussed. Recently, the Bezier curves, mainly the cubic curves (of third-degree), were used. The segments of the boundary shape were modeled by such curves (with ensuring continuity at the connection points). Using NURBS curves, the boundary shape can be modeled with only one curve. So, continuity is automatically ensured. Additionally, the second degree NURBS curve is enough to obtain the shape with high accuracy (better than cubic Bezier curves). The NURBS curve is defined by points, their weights and knots vector. Such parameters significantly improve the shape modification process, which can directly improve e.g. the shape identification process. To examine the impact of modeling accuracy on the final PIES solutions, examples described by the Navier-Lamé equations were used. To improve calculations, the PIES method using NURBS curves was implemented as a computer program. Then, it was decided to verify the accuracy of the obtained solutions. For comparison, the solutions were also obtained using analytical solutions, boundary element method, and PIES method (with the Bezier curves). An improvement in the boundary shape modeling was noticed. It significantly affects the accuracy of solutions. As a result, the consumption of computer resources was reduced, while the process of boundary shape modeling and the accuracy of the obtained results were improved.
\end{abstract}

Keywords: Boundary value problems $\cdot$ NURBS $\cdot$ BEM $\cdot$ PIES

\section{Introduction}

In practice, the boundary value problems can be defined with a very large variety of boundary shapes. It is very difficult to use analytical methods to solve such problems. Therefore, scientists started to use and develop numerical methods. 
Well known from the literature and widely used methods: finite element method (FEM) [3,7] and boundary element method (BEM) [2,8], use respectively the finite elements for modeling the domain or the boundary elements to define the boundary. Such methods tend to be less effective because, to improve the accuracy of modeling (what improves the solutions accuracy), the number of elements should be increased (what means greater consumption of computer resources).

In this paper, as an alternative to the classical discretization of the domain or boundary (occurring in the mentioned methods), a method of parametric integral equations system (PIES) was proposed $[4,9,10]$. This method uses the curves (known from computer graphics) directly included in the mathematical formalism of PIES. It makes modeling and modification of the shape much easier. Till now, in the PIES method, the Bézier curves were mainly used. These curves are defined using polynomial segments with an appropriate class of analytical and geometric continuity at their connection points.

With the development of computer graphics, the NURBS curves have appeared $[5,6]$. These curves give much more possibilities and significantly improve the accuracy of shape modeling. Therefore, they will be used for modeling the boundary shape of the boundary value problem solved by the PIES method. Now, the shape of the boundary can be modeled by one closed curve automatically (without additional care of the connection of segments). The definition of such a curve needs a set of points, weights, and knots. The point's weight determines its influence on the curve and makes it easier to accurately model curves such as the circle and ellipse. The knots allow to obtain corners and change the curve's degree. More information can be found in the paper about the NURBS curves in Laplace's equation [11]. Application of NURBS curves unifies the shape definition in PIES and also influences the modeling accuracy, which finally improves the accuracy of the solutions.

To highlight the benefits of using NURBS curves in PIES, the definition of the same shape for FEM, BEM, and PIES (using Bezier and NURBS curves) was presented on Fig. 1. Using NURBS curves here, it is enough to use two curves of the second degree (for inner and outer shape). Additionally, the 8 boundary points (4 for each shape) are enough to define properly such shape. Although the number of input data is reduced, the accuracy of the modeling is improved. So the solutions are also obtained with high accuracy.

\section{Inclusion of the NURBS Curves into PIES}

The general form of the PIES (for any shape of the boundary) for Navier-Lamé equation without mass forces is presented by the following formula [1]:

$$
0.5 \boldsymbol{u}_{l}\left(s_{1}\right)=\sum_{j=1}^{n} \int_{\widehat{s}_{j-1}}^{\widehat{s}_{j}}\left\{\boldsymbol{U}_{l j}^{*}\left(s_{1}, s\right) \boldsymbol{p}_{j}(s)-\boldsymbol{P}_{l j}^{*}\left(s_{1}, s\right) \boldsymbol{u}_{j}(s)\right\} J_{j}(s) d s,
$$

where $\widehat{s}_{l-1} \leq s_{1} \leq \widehat{s}_{l}, \widehat{s}_{j-1} \leq s \leq \widehat{s}_{j}$. 


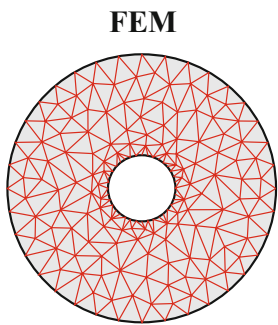

linear triangular finite element 310 elements 226 nodes
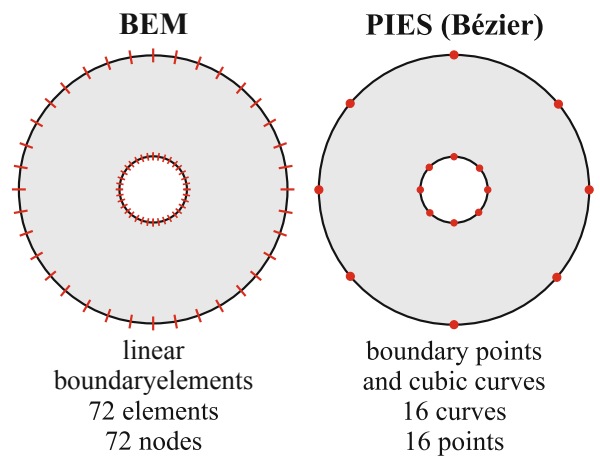

PIES (NURBS)

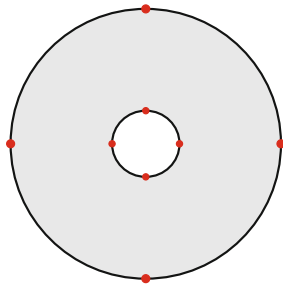

boundary points and quadratic curves 2 curves 8 points

Fig. 1. Comparison of modeling the shape of boundary for different methods.

Functions $\boldsymbol{p}_{j}(s)=\left[p_{j}^{(1)}(s), p_{j}^{(2)}(s)\right]$ and $\boldsymbol{u}_{j}(s)=\left[u_{j}^{(1)}(s), u_{j}^{(2)}(s)\right]$ are the boundary functions, that include the boundary condition. Function $J_{j}(s)$ is the Jacobian for segment of the curve $\boldsymbol{S}_{j}(s)$ and can be calculated as follows:

$$
J_{j}(s)=\left[\left(\frac{\partial S_{j}^{(1)}(s)}{\partial s}\right)^{2}+\left(\frac{\partial S_{j}^{(2)}(s)}{\partial s}\right)^{2}\right]^{0.5}
$$

where $S_{j}^{(1)}(s), S_{j}^{(2)}(s)$ are the vector components of a curve segment $\boldsymbol{S}_{j}=\left[S_{j}^{(1)}(s), S_{j}^{(2)}(s)\right]^{T}$, and depend on parameter $s$.

First integrand $\boldsymbol{U}_{l j}^{*}\left(s_{1}, s\right)$, for the plane state of strain, is defined as follows:

$$
\boldsymbol{U}_{l j}^{*}\left(s_{1}, s\right)=-\frac{1}{8 \pi(1-\nu) \mu}\left[\begin{array}{cc}
(3-4 \nu) \ln (\eta)-\frac{\eta_{1}^{2}}{\eta^{2}} & -\frac{\eta_{1} \eta_{2}}{\eta^{2}} \\
-\frac{\eta_{1} \eta_{2}}{\eta^{2}} & (3-4 \nu) \ln (\eta)-\frac{\eta_{2}^{2}}{\eta^{2}}
\end{array}\right],
$$

where $l, j=1,2, \ldots, n, \nu$ is the Poisson's ratio, $\mu$ is the Lamé constant (depends on the material constants). The shape of boundary is included by functions $\eta$, $\eta_{1}$ and $\eta_{2}$ defined as follows:

$$
\begin{gathered}
\eta=\left[\eta_{1}^{2}+\eta_{2}^{2}\right]^{0.5} \\
\eta_{1}=S_{l}^{(1)}\left(s_{1}\right)-S_{j}^{(1)}(s), \quad \eta_{2}=S_{l}^{(2)}\left(s_{1}\right)-S_{j}^{(2)}(s) .
\end{gathered}
$$

Second integrand $\boldsymbol{P}_{l j}^{*}\left(s_{1}, s\right)$ is described by formula:

$$
\boldsymbol{P}_{l j}^{*}\left(s_{1}, s\right)=-\frac{1}{4 \pi(1-\nu) \eta}\left[\begin{array}{ll}
P_{11} & P_{12} \\
P_{21} & P_{22}
\end{array}\right],
$$

where $l, j=1,2, \ldots, n$, and $\boldsymbol{P}_{i k}$ (where $\mathrm{i}=1,2$ and $\mathrm{k}=1,2$ ) are defined as follows:

$$
\boldsymbol{P}_{i i}=\left\{(1-2 \nu)+2 \frac{\eta_{i}^{2}}{\eta^{2}}\right\} \frac{\partial \eta}{\partial \boldsymbol{n}},
$$




$$
\begin{gathered}
\boldsymbol{P}_{i k}=\left\{2 \frac{\eta_{i} \eta_{k}}{\eta^{2}} \frac{\partial \eta}{\partial \boldsymbol{n}}-(1-2 \nu)\left[\frac{\eta_{i}}{\eta} n_{k}(s)+\frac{\eta_{k}}{\eta} n_{i}(s)\right]\right\} \\
\frac{\partial \eta}{\partial n}=\frac{\eta_{1}}{\eta} n_{1}(s)+\frac{\eta_{2}}{\eta} n_{2}(s) .
\end{gathered}
$$

where $n_{1}(s), n_{2}(s)$ are the components of the normal vector $\boldsymbol{n}_{j}$ to boundary segment $\boldsymbol{S}_{j}$.

Till now the curvilinear segments were defined by cubic Bézier curves. The equation describing corresponding segments of such a curve is presented as follows:

$$
\boldsymbol{S}_{m}(s)=\boldsymbol{V}_{0}(1-s)^{3}+\boldsymbol{V}_{1} 3(1-s)^{2}+\boldsymbol{V}_{2}(1-s) s^{2}+\boldsymbol{V}_{3} s^{3},
$$

where $m=l, j$. The shape of such curve is defined by four control points divided into: approximation points $\left(\boldsymbol{V}_{1}, \boldsymbol{V}_{2}\right)$ and interpolation points $\left(\boldsymbol{V}_{0}, \boldsymbol{V}_{3}\right)$.

The inclusion of the NURBS curves in PIES (1) requires in functions (5) the substitution of the following formula describing segments [6]:

$$
\boldsymbol{S}_{m}(s)=\frac{\sum_{i=0}^{n} w_{i} \boldsymbol{P}_{i} N_{i}^{k}(s)}{\sum_{i=0}^{n} w_{i} N_{i}^{k}(s)} \text { dla } t_{k} \leq s \leq t_{n+1},
$$

where $\boldsymbol{P}_{i}(i=0,1, \ldots, n)$ are the control points, $w_{i}(i=0,1, \ldots, n)$ are weights corresponding to points, and $N_{i}^{k}(s)$ is the function of k-degree, defined by the recursive formula:

$$
\begin{gathered}
N_{i}^{0}(s)= \begin{cases}1 & s \in\left\langle t_{i}, t_{i+1}\right) \\
0 & \text { otherwise }\end{cases} \\
N_{i}^{k}(s)=\frac{s-t_{i}}{t_{i+k}-t_{i}} N_{i}^{k-1}(s)+\frac{t_{i+k+1}-s}{t_{i+k+1}-t_{i+1}} N_{i+1}^{k-1}(s),
\end{gathered}
$$

where $0 \leq i \leq n-k-1,1 \leq k \leq n-1, \frac{0}{0}:=0$. The $t_{i}(i=0,1, \ldots, m+n+1)$ are the elements of the knot vector.

Additionally, to obtain values of Jacobian (2) and normal vector, the derivative with respect to parameter s of the NURBS curve function have to be calculated [6]:

$$
\frac{\partial \boldsymbol{S}_{m}(s)}{\partial s}=\frac{\sum_{i=0}^{n} w_{i} \boldsymbol{P}_{i}\left(N_{i}^{k}(s)\right)^{\prime} \sum_{i=0}^{n} w_{i} N_{i}^{k}(s)-\sum_{i=0}^{n} w_{i} \boldsymbol{P}_{i} N_{i}^{k}(s) \sum_{i=0}^{n} w_{i}\left(N_{i}^{k}(s)\right)^{\prime}}{\left(\sum_{i=0}^{n} w_{i} N_{i}^{k}(s)\right)^{2}}
$$

where $\left(N_{i}^{k}(s)\right)^{\prime}$ is defined as follow:

$$
\left(N_{i}^{k}(s)\right)^{\prime}=\frac{k}{t_{i+k}-t_{i}} N_{i}^{k-1}(s)-\frac{k}{t_{i+k+1}-t_{i+1}} N_{i+1}^{k-1}(s),
$$


The calculations processed using the above formulas cause significant computational time delay. Therefore, in the program, only NURBS curves of second degree were implemented. For calculation of the shape function and derivative, the analytical formulas were obtained (assuming the curve of the second degree).

\section{Verification and Advantages of NURBS Curves in PIES}

\section{Example 1 - Circular Hole}

First example is the circular hole with radius $R=10$. Inside the cylinder, defined in the plane state of strain, the force $p=15$ is applied. The material parameters are: $E=21$ and $\nu=0.1$. The shape modeling with Bézier curves, recently used in PIES, is presented in Fig. 2a. Only eight boundary points $P_{i}$ are shown, for better clarity (two additional approximation points should be defined between the boundary points in the cubic curve). For the same purpose, the one NURBS curve of the second degree (4 boundary points) is enough (Fig. 2b). Moreover, despite the smaller amount of input data, the NURBS curve modeled the shape more accurately than previously used cubic Bézier curves (the average radius over the entire circumference is respectively $R_{N}=9.9999$ and $\left.R_{B}=9.9944\right)$.

a)

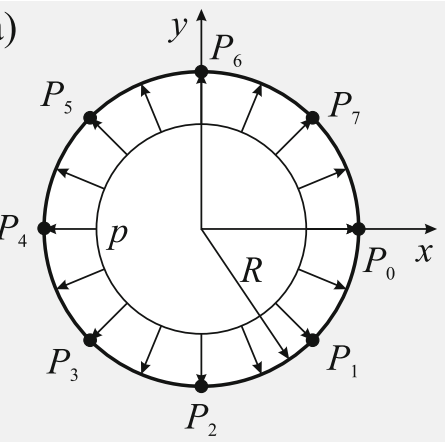

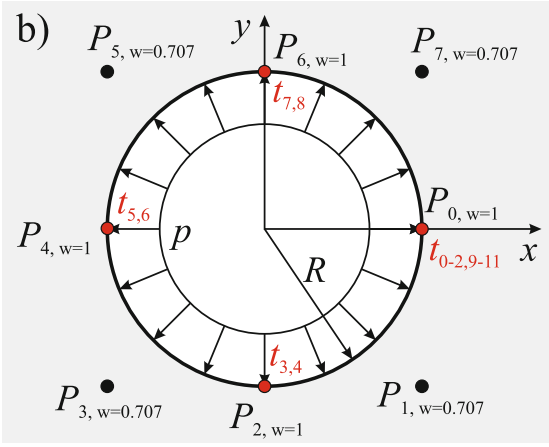

Fig. 2. Modeling the shape of boundary using a) Béziera, and b) NURBS, curves.

Solutions obtained using PIES (with NURBS curves) are compared with analytical [8] and numerical (BEM using 24 linear boundary elements [2] and PIES using Bézier curves) solutions in Table1. Comparing the PIES (with NURBS) solutions with analytical ones, the maximum average relative error $0.06 \%$ is obtained for $\sigma_{x}$. It is a significant improvement in comparison with PIES (using Bézier curves) or BEM, where the errors for $\sigma_{x}$ are respectively $0.37 \%$ and $2.04 \%$. 
Table 1. Solutions in the domain $(y=0)$ of boundary value problem from Fig. 2.

\begin{tabular}{|c|c|c|c|c|c|c|c|c|}
\hline \multirow[t]{2}{*}{$x$} & \multicolumn{2}{|c|}{ Analytical } & \multicolumn{2}{|c|}{ PIES (NURBS) } & \multicolumn{2}{|l|}{ BEM } & \multicolumn{2}{|c|}{ PIES (Bézier) } \\
\hline & $\sigma_{x}$ & $\sigma_{y}$ & $\sigma_{x}$ & $\sigma_{y}$ & $\sigma_{x}$ & $\sigma_{y}$ & $\sigma_{x}$ & $\sigma_{y}$ \\
\hline 12 & -10.417 & 10.417 & -10.426 & 10.415 & -10.222 & 10.222 & -10.359 & 10.421 \\
\hline 15 & -6.667 & 6.667 & -6.670 & 6.667 & -6.525 & 6.525 & -6.642 & 6.654 \\
\hline 20 & -3.75 & 3.75 & -3.751 & 3.750 & -3.67 & 3.67 & -3.742 & 3.743 \\
\hline \multicolumn{3}{|c|}{ Average relative error $[\%]$} & 0.06 & 0.01 & 2.04 & 2.04 & 0.37 & 0.14 \\
\hline
\end{tabular}

\subsection{Example 2 - Multiply Connected Domain}

The next example is the Lamé problem. The Fig. 3a presents only 16 boundary points of the shape modeled using Bézier curves. The additional 32 approximation points should be defined. Figure $3 \mathrm{~b}$ presents modeling using NURBS curves.
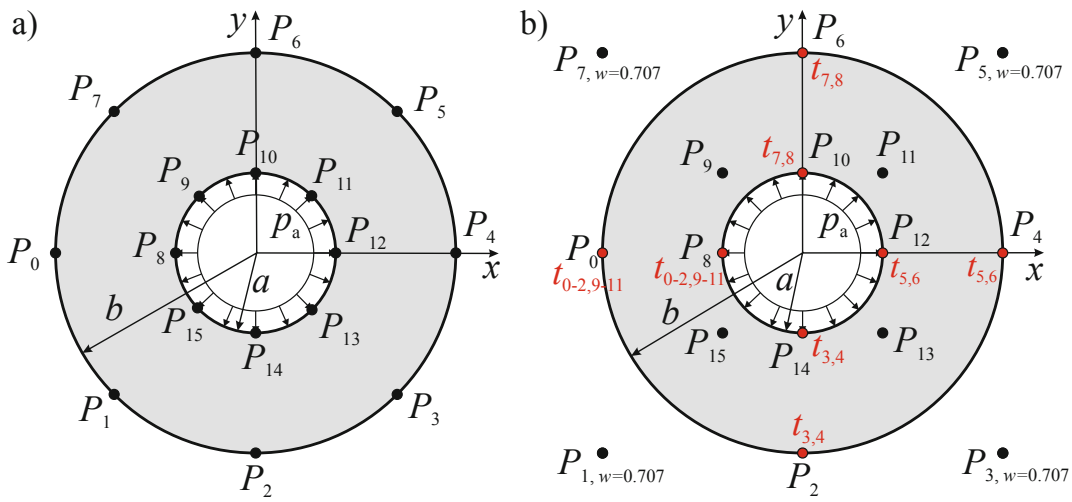

Fig. 3. Modeling the shape of boundary using a) Béziera, and b) NURBS, curves.

The cylindrical tube under inner hydrostatic pressure $p=100 M P a$ (in the plane state of strain) is considered. The inner radius is $a=10 \mathrm{~cm}$, and the outer is $b=25 \mathrm{~cm}$. The material parameters are: $E=2 * 10^{5} \mathrm{MPa}$ i $\nu=0.25$. Analytical solutions are defined as [8]:

$$
\sigma_{x}=\frac{p_{a} a^{2}-p_{b} b^{2}}{b^{2}-a^{2}}-\frac{\left(p_{a}-p_{b}\right) a^{2} b^{2}}{r^{2}\left(b^{2}-a^{2}\right)}, \quad \sigma_{y}=\frac{p_{a} a^{2}-p_{b} b^{2}}{b^{2}-a^{2}}+\frac{\left(p_{a}-p_{b}\right) a^{2} b^{2}}{r^{2}\left(b^{2}-a^{2}\right)}
$$

where $r$ is the one of polar coordinates and $r^{2}=x^{2}+y^{2}, a<r<b$. Analytical and PIES (with Bézier and NURBS curves) solutions are presented in Table 2. The example confirms the accuracy of the solutions using NURBS curves. 
Table 2. Comparison PIES solutions with analytical ones - example from Fig. 3.

\begin{tabular}{l|l|l|l|l|l|l|l|l|l|l}
\hline$x$ & \multicolumn{3}{l|}{ Analytical } & \multicolumn{2}{l|}{ PIES (Bézier) } & \multicolumn{2}{l|}{ Error [\%] } & \multicolumn{2}{l|}{ PIES (NURB) } & \multicolumn{2}{l}{ Error [\%] } \\
\cline { 2 - 13 } & $\sigma_{x}$ & $\sigma_{y}$ & $\sigma_{x}$ & $\sigma_{y}$ & $\sigma_{x}$ & $\sigma_{y}$ & $\sigma_{x}$ & $\sigma_{y}$ & $\sigma_{x}$ & $\sigma_{y}$ \\
\hline 12 & -63.624 & 101.720 & -63.220 & 101.811 & 0.635 & 0.089 & -63.681 & 101.681 & 0.090 & 0.038 \\
\hline 14 & -41.691 & 79.786 & -41.488 & 79.752 & 0.487 & 0.043 & -41.707 & 79.757 & 0.038 & 0.036 \\
\hline 16 & -27.455 & 65.551 & -27.364 & 65.535 & 0.331 & 0.024 & -27.467 & 65.529 & 0.044 & 0.034 \\
\hline 18 & -17.695 & 55.791 & -17.665 & 55.792 & 0.170 & 0.002 & -17.706 & 55.775 & 0.062 & 0.029 \\
\hline 20 & -10.714 & 48.810 & -10.724 & 48.803 & 0.093 & 0.014 & -10.724 & 48.798 & 0.093 & 0.025 \\
\hline 22 & -5.549 & 43.644 & -5.577 & 43.578 & 0.505 & 0.151 & -5.553 & 43.631 & 0.072 & 0.030 \\
\hline 24 & -1.620 & 39.716 & -1.547 & 39.500 & 4.506 & 0.544 & -1.569 & 39.695 & 3.148 & 0.053 \\
\hline \multicolumn{3}{l}{ Average relative error $[\%]:$} & & 0.96 & 0.12 & & & 0.51 & 0.03 \\
\hline
\end{tabular}

\section{Conclusions}

This paper presents the high efficiency of inclusion of NURBS curves in the PIES method and compare obtained solutions with the existing ones. Using NURBS curves, the number of points (necessary to model the boundary) is much lower. Using 8 cubic Bézier segments in modeling circle shape, the average radius over the entire circumference is $R=9.9944$ (expected $R=10$ ). However, using one NURBS curve of the second degree, the radius is $R=9.9999$. Therefore, using NURBS curves, the modeling accuracy increases, even with the lower number of points. Such an improvement is caused by two curve parameters: weights of points and knots. The shape modeling using NURBS in PIES is also more uniform. Till now, the curvilinear and linear segments were described using separate functions. Now, using NURBS curves, the shape will be described with one curve. The above examples present the advantages of modeling and its impact on improvement in the accuracy of obtained solutions. Therefore, using NURBS in PIES improves the process of modeling and modification of the boundary shape, significantly reduces the number of points (necessary to define the shape) and ultimately improves the accuracy of the solutions.

\section{References}

1. Boltuc, A., Zieniuk, E.: Modeling domains using Bezier surfaces in plane boundary problems defined by the Navier-Lame equation with body forces. Eng. Anal. Bound. Elem. 35(10), 1116-1122 (2011)

2. Brebbia, C.A., Telles, J.C.F., Wrobel, L.C.: Boundary Element Techniques: Theory and Applications in Engineering. Springer, New York (1984). https://doi.org/10. 1007/978-3-642-48860-3

3. Doitrand, A., Martin, E., Leguillon, D.: Numerical implementation of the coupled criterion: matched asymptotic and full finite element approaches. Finite Elem. Anal. Des. 168, 103344 (2020)

4. Kuzelewski, A., Zieniuk, E., Kapturczak, M.: Acceleration of integration in parametric integral equation system using CUDA. Comput. Struct. 152, 113-124 (2015) 
5. Noruzi, R., Ghadai, S., Bingol, O.R., Krishnamurthy, A., Ganapathysubramanian, B.: NURBS-based microstructure design for organic photovoltaics. Comput. Aided Des. 118, $102771(2020)$

6. Piegl, L.A., Tiller, W.: Computing the derivative of NURBS with respect to a knot. Comput. Aided Geom. Des. 15(9), 925-934 (1998)

7. Ji, S.Y., Wang, S.L.: A coupled discrete-finite element method for the ice-induced vibrations of a conical jacket platform with a GPU-based parallel algorithm. Int. J. Comput. Methods 17(4), 1850147 (2020)

8. Timoshenko, S.P., Goodier, J.N.: Theory of Elasticity. McGraw-Hill, Tokyo (1970)

9. Zieniuk, E., Szerszeń, K.: A separation between the boundary shape and the boundary functions in the parametric integral equation system for the $3 \mathrm{D}$ stokes equation. Numer. Algorithms 80(3), 753-780 (2018). https://doi.org/10.1007/s11075018-0505-3

10. Zieniuk, E., Kapturczak, M., Kużelewski, A.: Modification of interval arithmetic for modelling and solving uncertainly defined problems by interval parametric integral equations system. In: Shi, Y., Fu, H., Tian, Y., Krzhizhanovskaya, V.V., Lees, M.H., Dongarra, J., Sloot, P.M.A. (eds.) ICCS 2018. LNCS, vol. 10862, pp. 231240. Springer, Cham (2018). https://doi.org/10.1007/978-3-319-93713-7_19

11. Zieniuk, E., Kapturczak, M.: Modeling the shape of boundary using NURBS curves directly in modified boundary integral equations for Laplace's equation. Comput. Appl. Math. 37(4), 4835-4855 (2018) 